\title{
Allelic imbalance at the HER2/TOP2A locus in breast cancer
}

Cornelis J. J. Huijsmans ${ }^{1 *}$, Adriaan J. C. van den Brule ${ }^{1}$, Henny Rigter ${ }^{2}$, Jeroen Poodt ${ }^{1}$, Johannes C. van der Linden ${ }^{2}$, Paul H. M. Savelkoul ${ }^{3,4}$, Mirrian Hilbink ${ }^{5}$ and Mirjam H. A. Hermans ${ }^{1}$

\begin{abstract}
Background: Breast cancer is a heterogeneous disease with various histological features and molecular markers. These are utilized for the prediction of clinical outcome and therapeutic decision making. In addition to well established markers such as HER2 overexpression and estrogen and progesterone receptor (ER and PR) status, chromosomal instability is evolving as an important hallmark of cancers. The HER2/TOP2A locus is of great importance in breast cancer. The copy number variability at this locus has been proposed to be a marker for the degree of chromosomal instability. We therefore developed a Single Nucleotide Polymorphism (SNP) assay to evaluate allelic imbalance at the HER2/TOP2A locus in three different entities of primary breast tumors.

Methods: Eleven SNPs were carefully selected and detected by real time PCR using DNA extracted from paired (histologically normal and tumor) paraffin-embedded tissues. Primary breast tumors of 44 patients were included, 15 tumors with HER2 overexpression, 16 triple negative tumors, defined by the absence of HER2 overexpression and a negative ER and PR status and 13 ER and PR positive tumors without HER2 overexpression. As controls, histologically normal breast tissues from 10 patients with no breast tumor were included.
\end{abstract}

Results: Allelic imbalance was observed in 13/15 (87 \%) HER2 positive tumors, the remaining 2 being inconclusive. Of the 16 triple negative tumors, $12(75 \%)$ displayed instability, $3(19 \%)$ displayed no instability, and 1 was inconclusive. Of the 13 hormone receptor positive tumors, 5 (38\%) displayed allelic imbalance, while 8 did not.

Conclusions: We conclude that the SNP assay is suitable for rapid testing of allelic (im)balance at the HER2/TOP2A locus using paraffin-embedded tissues. Based on allelic imbalance at this locus, both triple negative and ER and PR positive breast tumors can be subcategorized. The clinical relevance of the allelic (im)balance status at the HER2/ TOP2A locus in breast cancer is subject of future study.

Virtual Slides: The virtual slide(s) for this article can be found here: http://www.diagnosticpathology.diagnomx.eu/ vs/2086062232155220

Keywords: Breast cancer, Allelic instability, HER2, TOP2A, Single nucleotide polymorphism

\section{Background}

Breast cancer is a heterogeneous disease that can display a diverse variety of biological features. Several proteins are well known to be expressed excessively in these tumors. Based on the excessive presence of such proteins, breast cancer can be divided in several different biological entities. This breast cancer categorization is

\footnotetext{
* Correspondence: r.huysmans@jbz.nl

'Laboratory of Molecular Diagnostics, Jeroen Bosch Hospital, PO Box 90153, 5200 ME 's-Hertogenbosch, The Netherlands

Full list of author information is available at the end of the article
}

utilized for the prediction of clinical outcome and therapy selection [1].

Well known examples of overexpressed proteins that play a critical role in breast cancer diagnostics and choice of therapy, are the estrogen and progesterone receptors (ER and PR). Available targeted therapies in these breast cancer cases are e.g. Tamoxifen and aromatase inhibitors [2-4].

In addition to the overexpression of ER and PR, aberrations of the chromosome 17q12-q22 genomic region play an important role in breast cancer. This locus incorporates the HER2 gene as well as the juxtaposed TOP2A gene, 
which is located $700 \mathrm{~kb}$ telomeric of HER2 [5-7]. The $H E R 2$ oncogene encodes the human epidermal growth factor receptor 2 protein, a $185 \mathrm{kDa}$ transmembrane tyrosine kinase receptor also known as erbB-2 and neu $[5,6]$. Upon ligand binding the other types of HER receptors will dimerize with HER2. Such dimerization triggers an integrate network of HER signal transduction involving a variety of signaling pathways [8-10]. When the HER2 oncogene is amplified, HER2 overexpression occurs resulting in overstimulation of HER mediated signal transduction [8]. HER2 is found to be amplified in 25$30 \%$ of breast cancers [11]. Patients with HER2 amplified breast tumors resulting in overexpression of HER2, benefit from treatment with the monoclonal antibody Trastuzamab (Herceptin). Trastuzamab is thought to abolish the HER2 mediated signaling of the HER signal transduction network [12, 13]. In addition, analysis of several studies has shown an association of improved adjuvant anthracycline (AC)-based chemotherapy response rates in HER2 amplified breast tumors [14]. However, there is no clear AC induced HER2 inhibiting mechanism, it is therefore suggested that TOP2A coamplification is the biological rationale behind these observations [15]. The TOP2A gene encodes topoisomerase II alpha, a $170 \mathrm{kDa}$ enzyme that catalyzes the topological DNA changes needed during the multistep process of cell division [16]. Coamplification of TOP2A occurs along with HER2 amplification in approximately 30 $45 \%$ of the HER 2 amplified cases [15, 17, 18]. Patients with TOP2A amplified breast tumors seem to benefit from adjuvant AC-based chemotherapy. ACs are chemotherapeutic agents that are known to have, among other mechanisms of action, TOP2A inhibiting properties. TOP2A catalytic activity is inhibited by ACs via stabilization of the intermediate complex where TOP2A tyrosine residues are covalently linked to the 5' phosphoryl ends of the cut DNA strands during the topological changes which they catalyze. Trapping the complex in this state prevents DNA resealing and further cell cycle progress, which ultimately induces programmed cell death $[16,19,20]$. In patients that are treated with AC-based chemotherapy, breast tumors with TOP $2 A$ amplification show a trend towards a better disease free survival than tumors lacking this amplification. In addition, TOP $2 A$ amplification is an independent predictor of a significantly higher pathological complete response rate in these patients [18]. On the contrary, TOP $2 A$ deletion, occurring in $15-40 \%$ of HER2 amplified cases, has been associated with AC resistance $[15,17,18]$. Tumors lacking ER and PR overexpression as well as HER2 amplification are defined as "triple negative" breast tumors. Triple negative tumors are associated with poor clinical outcome and lack of (currently) available targeted therapy [21, 22].
The above mentioned biological characteristics are well established biomarkers utilized to categorize breast cancer types for diagnostic and treatment related purposes. More recently, high resolution array based systems, targeting Single Nucleotide Polymorphisms (SNPs) or utilizing Comparative Genomic Hybridization, have been employed to subcategorize these breast tumor types and revealed distinct profiles of gene-expression or genetic alterations [2328]. Hormone receptor positive tumors without HER2 overexpression showed minor and less frequent chromosomal instability in comparison to HER2 overexpressed and triple negative tumors that showed more extensive and more frequent chromosomal instability [23-29]. Copy number alterations across the genomic region incorporating the HER2/TOP2A locus have been proposed to be a marker for the degree of chromosomal instability in breast cancer [30,31]. Early breast cancer patients with a tumor containing extensive chromosomal instability, that were treated with AC-based therapy, were shown to have a decreased risk of death and a trend towards a decreased risk of relapse. Similarly treated patients with a tumor of low chromosomal instability showed no apparent benefit [30, 31]. Determining such instability in a diagnostic setting may therefore be of clinical relevance as an adjuvant molecular marker.

Single Nucleotide Polymorphisms (SNP) are easily detected by real time PCR, a technique that is widely available in many laboratories that routinely employ molecular diagnostics. By analyzing a panel of heterozygous SNPs, copy number alterations and thereby allelic imbalance of the involved locus can be determined. It therefore may provide an accessible method to assess allelic imbalance at the $H E R 2 / T O P 2 A$ locus.

\section{Methods}

\section{Study population and clinical specimens}

The study was carried out retrospectively with the approval of the Board of Directors and the Scientific Advisory Board of the Jeroen Bosch Hospital. All patient samples were rendered anonymous before use in this study.

\section{Control group}

Ten histologically normal formalin-fixed paraffin-embedded (FFPE) breast -tissues from 10 different patients (CT1-10) with no breast tumor were included as controls (Additional file 1: Table S1). Age of included patients was $32.8 \pm$ 12.0 years (mean age \pm 1 standard deviation (STD)).

\section{Breast tumor patient group}

Breast tumors (FFPE tissue) with known HER2 immunohistochemistry (IHC) and silver in situ hybridization (SISH) status from 44 patients were included in this study (Additional file 2: Table S2): 15 tissues with HER2 amplification (IHC 3+, patients HER2 +1-15; aged 52.9 \pm 
14.2 years (mean age \pm 1 STD)) 16 triple negatives (patients TN1-16; aged $53.4 \pm 12.9$ years (mean age \pm 1 STD)) and $13 \mathrm{ER} / \mathrm{PR}$ positive and HER2 non-amplified (ERPR+) breast tumors (patients ERPR $+1-13$; aged $55.5 \pm 9.3$ years (mean age \pm 1 STD)). To establish the germline SNP profile, a histologically normal archival FFPE tissue (breast tissue or lymph node tissue in case no breast tissue was available; Additional file 2: Table S2) from each patient was analyzed.

\section{Tissue Processing}

Tissues were fixed and processed as previously described [32]. Three- $\mu M$-thick sections were cut from each FFPE tissue and mounted on glass slides in sevenfold. One was used for Hematoxylin \& Eosin (HE) staining, two to four (depending on tissue size, with a total of approximately $2 \mathrm{~cm}^{2}$ ) for genomic DNA extraction, one for SISH and one for IHC. The latter two were only performed for the tumor samples.

\section{HER2 immunohistochemistry}

To establish the HER2 expression status, IHC was performed on all 44 tumor samples using the Ventana antiHer2/neu(4B5) (Roche Diagnostics GmbH, Mannheim, Germany) in combination with the VENTANA system (Roche Diagnostics $\mathrm{GmbH}$ ) according to the manufacturers protocol. IHC 0 and $1+$ were considered to represent normal expression, 2+ was considered borderline with regard to expression and 3+ was considered to represent overexpression.

\section{HER2 silver in situ hybridization}

To determine the HER2 amplification status and to assess chromosome 17 copy number status, SISH was performed on all 44 tumor samples using the INFORM HER2 Dual colour SISH DNA probe cocktail (Roche Diagnostics $\mathrm{GmbH}$ ) in combination with the VENTANA system (Roche Diagnostics $\mathrm{GmbH}$ ) according to the manufacturers protocol. Twenty nuclei were counted independently by 2 individuals. The mean from these 2 nuclei counts was calculated. Interpretation was as follows: a ratio (HER2 probe/centromere probe) of $<1.8$ represented no amplification; a ratio of 1.8-2.2 was equivocal and a ratio of $>2.2$ represented amplification.

\section{Genomic DNA extraction from FFPE tissues}

From each FFPE tissue, tumor as well as (reference) histologically normal tissue, a HE-slide was made. The tumor tissue containing HE slides were used to determine the area with the highest tumor cell percentage (TC\%). The HE slides containing the histologically normal tissue were used to determine the location of the highest nuclei density. Subsequently, these were used as a reference for tumor and histologically normal cell macrodissection, respectively. The respective cells were scraped off the corresponding blank slides. The scrapings were used for digestion and subsequent EasyMAG NucliSens (BioMérieux Benelux BV, Zaltbommel, The Netherlands) DNA extraction as described previously [32].

\section{SNP selection}

From the TaqMan ${ }^{\circ}$ SNP Genotyping Assays from Applied Biosystems, 11 suitable SNPs were chosen using SNP browser software Version 4.0 (Applied Biosystems, Table 1). SNP 1-6 were located up- and downstream of the HER2 gene within the HER2 smallest region of amplification [33]. The remaining 5 SNPs $(7-11)$ were located up- and downstream of the TOP $2 A$ gene. The selected SNPs displayed an allele frequency of approximately 0.5 in Caucasian (CEU, CEPH (Centre d'Etude du Polymorphisme Humain) from Utah), Chinese (CHB, Chinese from Beijing), Japanese (JPT, Japanese from Tokyo) and African (YRI, Yoruba from Ibadan Nigeria) populations. The latter to maximize the chance for heterozygosity, independent of the patients' ethnicity, for only those SNPs are informative.

\section{Allelic instability testing by SNP detection}

For real time PCR based amplification and detection of the selected SNPs, corresponding predesigned TaqMan ${ }^{\circ}$ SNP Genotyping Assays were purchased (Applied Biosystems, Foster City CA, USA; Table 1) containing two primers and two MGB TaqMan probes (5' VIC for allele

Table 1 Panel of selected Single Nucleotide Polymorphisms (SNPS)

\begin{tabular}{lrllllllll}
\hline SNP & dbSNP-ID & SNP-ID & Base (17q) & \multicolumn{3}{l}{ SNP } & \multicolumn{3}{l}{ Minor allele frequency } \\
$\mathrm{nr}$ & (rs) & (hCV) & & & CEU & CHB & JPT & YRI \\
\hline 1 & 7214151 & 30187206 & $34,761,298$ & C/T & 0.38 & 0.04 & 0.45 & 0.46 \\
2 & 7503195 & 437172 & $34,976,041$ & A/G & 0.38 & 0.04 & 0.45 & 0.45 \\
3 & 876493 & 7452397 & $35,078,071$ & G/A & 0.36 & 0.23 & 0.43 & 0.43 \\
4 & 4795408 & 30547514 & $35,361,153$ & A/G & 0.47 & 0.43 & 0.47 & 0.39 \\
5 & 4794822 & 31651885 & $35,410,238$ & T/C & 0.42 & 0.38 & 0.39 & 0.39 \\
6 & 868150 & 471861 & $35,466,885$ & A/G & 0.42 & 0.34 & 0.44 & 0.45 \\
7 & 2015561 & 7479296 & $35,846,755$ & G/A & 0.46 & 0.44 & 0.42 & 0.00 \\
8 & 4890114 & 27932396 & $35,857,075$ & T/C & 0.46 & 0.44 & 0.42 & 0.00 \\
9 & 8065040 & 11876205 & $36,073,767$ & A/G & 0.37 & 0.43 & 0.43 & 0.36 \\
10 & 10491123 & 29903401 & $36,086,722$ & A/G & 0.37 & 0.44 & 0.44 & 0.47 \\
11 & 7502428 & 29885409 & $36,133,740$ & C/T & 0.47 & 0.44 & 0.43 & 0.41 \\
\hline
\end{tabular}

SNP-ID $(\mathrm{rs})=$ reference SNP ID number. SNP-ID $(\mathrm{hCV})=$ Celera SNP ID. Base $(17 q)=$ the nucleotide position on chromosome 17(q). The HER2 gene is positioned at base 35,097,919-35,138,441, the TOP2A gene at 35,798,32135,827,695 (SNP browser software Version 4.0, Applied Biosystems). SNP 1-6 were located up- and downstream of the HER2 gene within the HER2 smallest region of amplification [33]. The remaining 5 SNPs (7-11) were located up- and downstream of the TOP2A gene. The particular nucleotide variation is referenced in "SNP"-column. Minor allele frequencies are indicated for different populations: CEU, CEPH (Centre d'Etude du Polymorphisme Humain) from Utah; CHB, Chinese from Beijing; JPT, Japanse from Tokyo and YRI, Yoruba from Ibadan Nigeria 
1, 5' FAM for allele 2 and a 3' black hole quencher for both alleles). The SNP assays were used according to the manufacturer's instructions. Real time PCR based SNP detection was performed as previously described [32], in an ABI Prism 7500 FAST SDS (Applied Biosystems) machine for $1 \mathrm{~min}$ at $95^{\circ} \mathrm{C}$, followed by 45 cycles of $3 \mathrm{~s}$ at $95^{\circ} \mathrm{C}$ and $30 \mathrm{~s}$ at $60^{\circ} \mathrm{C}$. To maximize pipetting accuracy and thereby minimize its influence on the data generated, all initial PCR setups were performed using the Hamilton StarLet instrument (Hamilton Robotics, Bonaduz, Switzerland).

Subsequent analysis was based on the generated fluorescence emission intensity of the VIC and FAM reporter dyes divided by the fluorescence emission intensity of the passive reference ROX dye (delta $\mathrm{Rn}$ value). When both alleles were present, a delta Rn VIC/FAM ratio could be established (allele 1/allele 2). This ratio was considered to be informative with regard to the relative number of alleles present and therefore a reflection of the allelic (im)balance status. Only the SNPs that were heterozygous for a given patient were informative. A schematic representation of this experimental scheme is presented in Fig. 1.

For each informative SNP the VIC/FAM ratios were plotted in a scatterplot. To minimize the influence of DNA concentration on VIC/FAM ratio and therefore data interpretation, the delta Rn FAM was corrected. The correction was performed by adapting the delta Rn FAM values of the histologically normal samples per SNP according to the

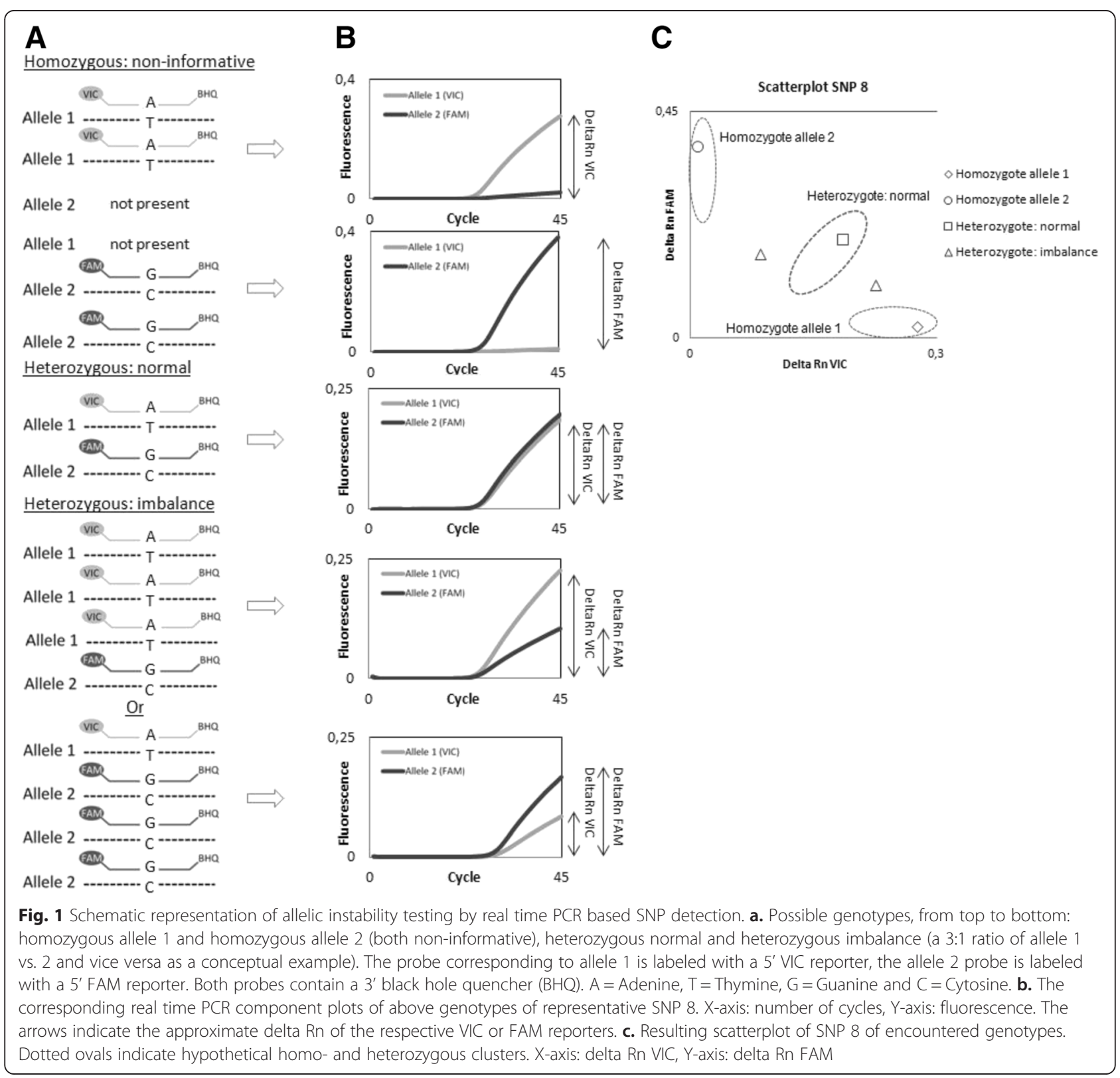


crossing point of the trend line with the y-axis. This resulted in the translocation of the trend lines of the histologically normal tissues so that they all passed through the origin. All delta Rn FAM data of the tumor samples were subsequently corrected accordingly.

\section{Statistical analysis allelic instability testing}

To gain insight into the distribution of delta Rn VIC/FAM ratios (ratio allele 1 /allele 2 ), the $25^{\text {th }}$ and $75^{\text {th }}$ percentiles of the ratios generated by all histologically normal tissues (from the control group as well as the group of breast tumor patients) were calculated for each SNP assay using Microsoft Office Excel 2007 (Microsoft, Redmond WA, USA). The distance between these percentiles represents the interquartile range (IQR), which was used to establish cut-off values for the normal ratio distribution per SNP. The +1.5 IQR and $-1.5 \mathrm{IQR}$ were taken as cut-off value for normal ratio distribution for 10/11 SNPs (all SNPs except SNP 3). All ratios with an IQR of $<1.5$ follow normal distribution and were interpreted as genetically stable with regard to the HER2/TOP2A locus. Ratios plotted within $1.5-3 \times \mathrm{IQR}$ were considered equivocal with regard to $H E R 2 / T O P 2 A$ allelic instability status. Ratios plotted $\geq 3 \mathrm{x}$ IQR were considered to be representative for HER2/ TOP2A allelic instability. Due to the large IQR generated using SNP 3 (varying from 2.3 to 15.1 times as large as found with the other SNPs), the cut-off values for that particular SNP were defined as follows: normal $=-1$ to +1 $\mathrm{IQR}$; equivocal $=1-2 \mathrm{IQR}$ and $\geq 2 \mathrm{IQR}=$ indicative of allelic instability.

When a sample generated an equivocal result for any of the employed SNPs, the analysis of that particular sample was repeated in duplicate (with non-diluted DNA and with 5-fold diluted DNA in Tris Low EDTA buffer) using the respective SNP. When $2 / 3$ results corresponded, the sample was called accordingly (e.g. equivocal/equivocal/ normal was called equivocal). When results were conflicting, the sample was called equivocal (e.g. equivocal/allelic instability/normal).

\section{Results}

Two patient groups were used in this study: a control group of 10 histologically normal tissues from patients (CT1-10) with no breast tumors and a group of 44 breast tumor patients with different entities of primary breast cancer (HER2 + 1-15, TN1-16 and ERPR+ 1-13) of which both tumor and normal tissues were available.

\section{SNP selection}

Technical validation of the selected SNP assays was performed using the 10 histologically normal breast tissues from the control group. All SNP assays had a sufficient sensitivity, yielding cycle-threshold values of $<35$ (threshold 0.007), a cut-off above which results are routinely considered less reliable in our laboratory. In addition, little or no cross-reaction of the SNP assays' allele 1 corresponding probes (VIC reporter) with allele 2, and of the allele 2 corresponding probes (FAM) with allele 1 was observed. This resulted in clear clustering of homozygous allele 1 , homozygous allele 2 and heterozygous patients. The panel of 11 SNP markers (Table 1) was therefore used to analyze the clinical specimens (Additional file 1: Table S1 and Additional file 2: Table S2).

\section{SNP analysis of patient materials}

To minimize the influence of DNA concentration on VIC/ FAM ratio and therefore data interpretation, a correction on all delta Rn FAM data was performed as described in the Materials and Methods section. Fig. 2 shows the corrected scatterplot of representative SNP 8 for histologically normal tissues from the control group and paired histologically normal and tumor samples of all patients in the breast tumor group. Ratios of each sample pair (histologically normal and tumor tissues) were connected.

Subsequently, the delta Rn VIC/FAM ratios of all informative SNPs of the histologically normal tissues from both groups (10 tissues from the control groups and 44 tissues from the breast tumor group) were used to gain insight in normal data distribution. Combining the data of the histologically normal tissues from these groups was justified due to the mutual similarity of delta Rn VIC/FAM ratio distribution amongst both groups. To determine the HER2/ $T O P 2 A$ allelic (im)balance, fluorescent ratios of the panel of 11 informative SNP's were analyzed using the cut-off values based on the interquartile range of data distribution amongst the histologically normal samples.

\section{Histologically normal tissues}

As expected, all informative SNP results (total of 54 SNPs of 10 patients) of the breast tissues from the control group fell within the normal IQR range ( -1.5 to $1.5 \mathrm{IQR}$ for SNP 1, 2 and 4-11 and -1 to 1 IQR for SNP 3). Overall results are depicted in Additional file 1: Table S1, Fig. 2 represents the corrected scatterplot of representative SNP 8.

The 44 histologically normal samples of the breast tumor patient group generated SNP results that fell within the normal IQR range and are therefore in agreement with the normal range observed in the control group. Only 1 out of the total of 208 informative SNPs yielded an equivocal result (patient TN2, SNP 11, triplicate analysis yielded 2 equivocal results and 1 result was found to indicate allelic instability).

\section{Breast tumor tissues}

Results of IHC and SISH are depicted in Additional file 2: Table S2. The SNP assays were used to investigate in more detail the genomic composition of the HER2/TOP2A locus. The results are summarized in Additional file 2: Table S2. 


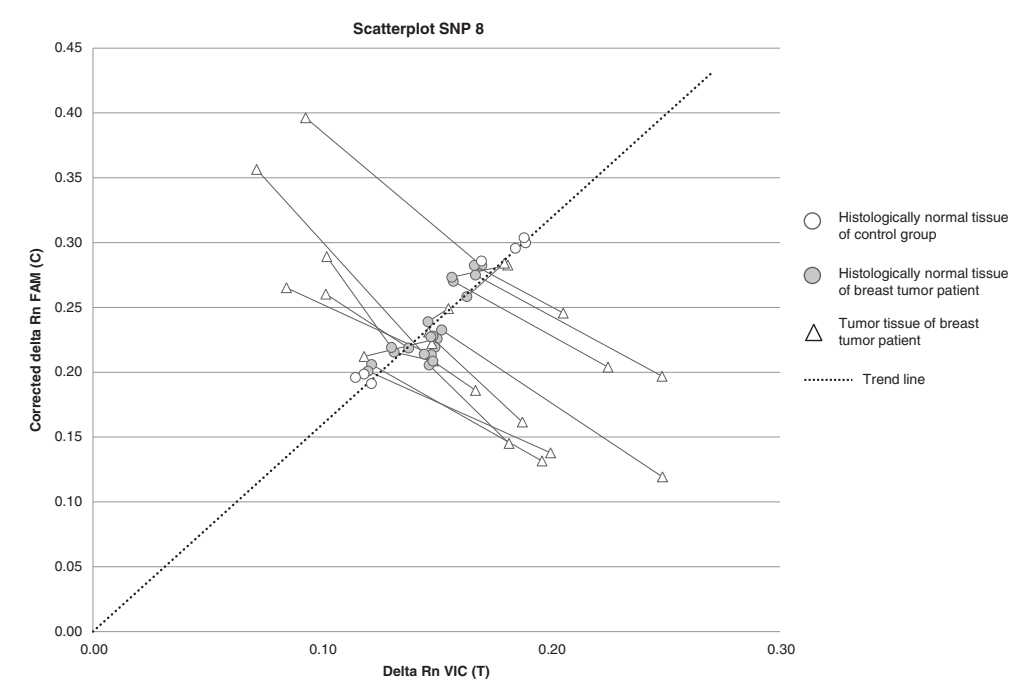

Fig. 2 Visualization of VIC/FAM ratios that were generated using representative SNP 8 after FAM correction. Scatterplot of SNP 8 analyses of histologically normal tissues from the control group and paired histologically normal and tumor tissues from the group of breast tumor patients. Connected symbols indicate normal and tumor tissue of a single patient. X-axis: delta Rn VIC ( $T=$ Thymine), Y-axis: corrected delta Rn FAM (C= Cytosine)

\section{HER2+ group}

Within the group of patients with tumors overexpressing HER2, the SNP data of patients HER2+ 2-5 and 7-15 were consistently indicative of an instable HER2/TOP2A locus. IHC scores were 3+, SISH ratios were 2.0-15.2 and $\mathrm{TC} \%$ ranged from 40 to $80 \%$ SNP 1 in patient HER2+ 8 generated an equivocal result. SNP results from patient HER2+ 1 and 6 showed inconsistent results between informative SNPs. All informative SNPs were therefore subsequently repeated in duplicate for these patients yielding identical results. SNP results from patient HER2+ 1 showed allelic balance of SNPs 8 and 9, whereas results from SNPs 1 and 10 were found to indicate allelic instability. IHC yielded a 3+ score, whilst the SISH ratio was 1.1. The sample had a TC\% of $30 \%$. SNP results from patient HER2+ 6 were also inconsistent. SNPs 1, 2 and 8 were indicative of allelic stability, while results from SNPs 4, 7 and 11 showed allelic instability. $\mathrm{IHC}, \mathrm{SISH}$ ratio and $\mathrm{TC} \%$ in this patient were $3+, 4.1$ and $80 \%$, respectively.

\section{Triple negative group}

In the triple negative group, patients TN1-4, 6, 9, 10 and 12-16 showed SNP results consistent with instability of the HER2/TOP2A locus. IHC scores, SISH ratios and TC\% varied from $0-2+, 0.7-1.9$ and $25-90 \%$, respectively. Several SNPs generated equivocal results in patients TN1 (SNP 3 3x equivocal), 3 (SNP 4 3x equivocal), 8 (SNP $12 x$ equivocal, $1 x$ normal and SNP $23 x$ equivocal), 10 (SNP $32 x$ equivocal, $1 x$ allelic instability) and 13 (SNP 1 and 3 both 3x equivocal). Patients TN5, 7 and 11 revealed no signs of HER2/TOP2A allelic imbalance, IHC scores were $0-1+$, SISH yielded ratios of
1.1-1.6 and the samples contained 30-95 \% tumor cells. In patient TN8, the 2 SNPs that were informative (SNPs 1 and 2) yielded equivocal results. IHC score, SISH ratio and $\mathrm{TC} \%$ for this patient were $1+, 0.9$ and $70 \%$, respectively.

\section{Estrogen and progesterone positive group}

From the ERPR+ group, patients ERPR $+1-3,6$ and 7 yielded SNP results indicative of $H E R 2 / T O P 2 A$ locus instability. IHC scores, SISH ratios and TC\% varied from $0-1+, 0.5-1.1$ and $30-70 \%$, respectively. Equivocal results were generated in patients ERPR+ 3 (SNP $33 x$ equivocal) and 7 (SNP $31 \mathrm{x}$ equivocal, $1 \mathrm{x}$ allelic instability and $1 \mathrm{x}$ normal). The ratio of SNP 3 was found to be equivocal (equivocal, allelic instability and normal) in patient ERPR + 7. The breast tumor tissues from patients ERPR + 4, 5 and 8-13 showed a stable HER2/TOP2A locus, IHC scores were $0-1+$, SISH ratios ranged from $1.0-1.5$ and a TC\% was $50-70 \%$. Patient ERPR +8 and 11 generated an equivocal result utilizing SNP 8 ( $2 x$ equivocal, $1 \mathrm{x}$ allelic instability) and 4 (3x equivocal), respectively.

\section{Discussion}

We have shown that the presented SNP based real time PCR assay is easy to perform and can be employed to reveal allelic instability of the HER2/TOP2A locus in breast tissues. The assay yielded results that fell within the normal range of data distribution in the control tissues. SNP results were consistent (all informative SNPs stable or instable) within most (42/44) tumor samples and were indicative of abundant allelic imbalance in HER2 amplified and TN tissues. 
In all patients (15/15) where HER2 overexpression was observed by IHC, SNP results were found to be indicative of $H E R 2 / T O P 2 A$ allelic instability. In 2 patients conflicting results between informative SNPs were found. In one sample (patient HER2+ 1), SNP 1 (instable in $2 / 3$ observations and 1 equivocal) and SNP 10 results showed allelic instability while no allelic instability was observed for SNP 8 and 9. SISH analysis of this sample revealed no genomic amplification of the HER2 locus. This possibly implies a false positive IHC result or HER2 overexpession not related to gene amplification, which has been reported in 3-8 \% of breast tumors [34-36]. However, the SNP assay did show the presence of a genetic aberration of part of the locus under investigation. Additionally, in patient HER2+ 6, allelic instability was observed for 3 non-contiguous SNPs out of 6 informative SNPs. The other 3 non-contiguous SNPs showed results indicative of a stable HER2/TOP2A locus. These observations may be the result of a combination of relatively small genetic aberrations each affecting part of the locus.

In the TN group, $75 \%$ of the tumor samples $(12 / 16)$ showed results indicative of allelic instability of the HER2/ TOP2A locus. This is in line with previous findings where the complex genomic profiles, with numerous gains and losses, of these tumors were classified as "sawtooth patterns" [23-28]. Nineteen percent of the TN patients (3/16) yielded results that were representative of a stable HER2/ TOP2A locus. In one sample (patient TN8) the only 2 informative SNPs were found to be equivocal, making the overall result with regard to the stability of the HER2/ TOP2A locus equivocal.

Within the ERPR+ group, $38 \%$ of the tumor samples $(5 / 13)$ yielded results representative of allelic instability of the HER2/TOP2A locus, whilst $62 \%$ (8/13) did not. Patient ERPR +7 showed discordant results in SNP 3, which was therefore concluded to be equivocal. Although the biological and/or technical rationale behind this observation remains unclear, we believe that it is justified to conclude that this sample harbors HER2/ TOP2A allelic instability due the data of the remaining 5 informative SNPs. Beside the HER2 normal tissue, patient ERPR + 11 also harbored HER2 amplified tumor tissue, which was most likely caused by the presence of a second tumor or tumor heterogeneity. The tissue included in this study, which had a stable HER2/TOP2A locus, was not HER2 amplified and was ERPR positive. This patient was therefore included in the ERPR+ group.

High chromosome instability, detected using FISH, in breast tumors is of prognostic value with regard to clinical outcome and may be useful in predicting response to AC-based therapy [31], since topoisomerase is targeted by AC. It is of particular interest whether these observations are also true for the allelically imbalanced groups found amongst the TN and the ERPR+ patient groups using the presented SNP based assay. Although the number of samples in our study is limited, the portion of tumor samples that contain HER2/TOP2A allelic instability in the TN and ERPR+ group $(75 \%$ and $38 \%$, respectively) roughly corresponded to the AC-based chemotherapy response rates found within these groups ( $85 \%$ and $47 \%$, respectively) [37]. A future study analyzing patients with available 5 year clinical follow-up data is needed to support this observation.

Overall, 8 out of 10 tissues where part of the tumor cells harbored polysomy of chromosome 17 , which was detected by the chromosome 17 centromeric probe included in the SISH assay, showed SNP results indicative of allelic instability. The SNP observations however may be the cumulative effect of several genetic aberrations including the polysomia. Reflection of monosomy and trisomy of chromosome 17 in the SNP results was less evident. In all tumors where aneuploidy chromosome 17 was observed, heterogeneity was observed with regard to chromosome 17 status as well.

SNP 3 cut-off values were adapted due to its large normal ratio distribution. Employing identically calculated cut-off values for SNP 3 would lead to the unjustified normal/equivocal calling of samples that clearly demonstrate a deviant VIC/FAM delta Rn ratio based on the generated scatterplot (data not shown).

In the histologically normal tissues, $99.5 \%$ (207/208) of the informative SNPs yielded results indicative of a stable HER2/TOP2A locus. Since sample input of these tissues was similar to those of the tumor tissues, PCR inhibition and depletion of reagents, which may occur, do not seem to be of major impact in allelic stability vs instability calling. The same is true, after the correction of delta $\mathrm{Rn}$ FAM values as described earlier, for variation in DNA concentration. The single anomaly concerning SNP 11 that was found aberrant in a histologically normal sample from the breast tumor group may be caused by a small deletion, point mutation(s) or a small recombination event around the involved SNP. Although sequencing of this SNP locus of this particular patient is of interest, it is beyond the scope of this study. Although the SNP test performed well, some drawbacks may be encountered. When analyzing a heterogeneous tumor tissue, unjustified (im)balance calling may occur. The resolution per SNP is relatively low, which may lead to an inconclusive result when (a large part of) the employed SNPs are homozygous and therefore non-informative. However, this issue is easily overcome by increasing the number of SNPs to be analysed. Determining the allelic instability of a sample with the SNP assay, as described here, costs approximately $€ 50-€ 75$ (US \$55-85) per sample. These costs are comparable to those of FISH based allelic instability testing. Additionally, the SNP test is well suited for the detection of copy number variations, but is not able to distinguish 
between various underlying mechanisms (e.g. gains vs. losses). SNP assays have a limited sensitivity, around $20 \%$ "mutant" DNA extracted from whole blood is detectable in a "wildtype" background [32]. For FFPE tissues, this sensitivity might be even somewhat lower. Therefore, to prevent false negativity with regard to allelic instability, the minimum tumor cell percentage was set at $25 \%$. This implies that allelic imbalance in minor tumor cell populations may have stayed unnoticed. Unfortunately, further analysis of the assays' sensitivity was not possible due the lack of quantified DNA affected by allelic instability. However, we do not expect DNA degradation to have a significant impact on the assays' sensitivity because of the small amplicons targeted (up to $80 \mathrm{bp}$ ) in combination with the size of amplifiable DNA fragments yielded by the employed DNA extraction method [38].

To minimize the effect of pipetting inaccuracy and to prevent pipetting errors, manual liquid handling was reduced to a minimum by performing all initial PCR setups using a Hamilton StarLet robot. Any inaccuracies or errors with regard to pipetting could negatively influence the determination of the natural ratio distribution of the histologically normal tissues and ultimately the allelic stability vs. instability calling.

\section{Conclusions}

In summary, the presented SNP methodology is easy to perform when compared to other copy number variation detection techniques such as array based systems targeting SNPs and Comparative Genomic Hybridization and corresponds well to positive SISH results. The real time PCR based SNP test may have an added value in the TN as well as the ERPR+ patient group. Within these groups, $75 \%$ of the TN and $38 \%$ of the ERPR+ tumors showed allelic instability with the SNP based assay. To determine such a possible added value of the presented SNP test a large-scale study should be performed. Ample size TN and ERPR+ patient groups with 5 year clinical follow-up data should be included to assess whether the determination of allelic instability of the HER2/TOP2A locus as a marker has clinical relevance and is of any significance for therapeutic decision making.

\section{Additional files}

Additional file 1: Table S1. Patient data and SNP test results from the control group. Patient data and SNP results. The top right represents the HER2/TOP2A locus and the schematic positions of the employed SNP markers. The arrows indicate the location of the HER2 and TOP2A genes. The boxes represent all informative SNPs that were considered not affected (green)/affected (red) by allelic instability or equivocal (grey). Homozygous SNPs are not informative, and are therefore shown as a horizontal line.

Additional file 2: Table S2. Patient data, tumor characteristics and IHC, SISH and SNP test results from breast tumor group. Patient data, tumor characteristics and $I \mathrm{HC}$, SISH and SNP test results. $\mathrm{L}$ and $\mathrm{B}$ represent the use of lymph node or breast histologically normal tissue. $\mid \mathrm{HC}=$ immunohistochemistry, SISH = silver in situ hybridization, HER2 $+=$ HER2 overexpression observed based on $\mathrm{IHC}$, TN = triple negative, ERPR+ = estrogen receptor and progesterone receptor positive. Chr17 status = chromosome 17 status with M (monosomy), $\mathrm{D}$ (disomy), T (trisomy) and P (polysomy) as possible outcomes supplemented with the number of chromosomes 17 observed per nucleus. Pred. $=$ predominantly and $\mathrm{TC} \%=$ tumor cell percentage. Failed $=\mathrm{SISH}$ repeatedly failed. The top right represents the HER2/TOP2A locus and the schematic positions of the employed SNP markers. The arrows indicate the location of the HER2 and TOP2A genes. The boxes represent all informative SNPs that were considered not affected (green)/affected (red) by allelic instability or equivocal (grey). Homozygous SNPs are not informative, and are therefore shown as a horizontal line.

\section{Competing interests}

The authors declare that they have no competing interests.

\section{Authors' contributions}

$\mathrm{CJ} \mathrm{H}$ participated in the design of the study, carried out molecular experiments, performed statistical analyses and drafted the manuscript. AJCB participated in the design of the study and drafted the manuscript. HR performed collection and pre-analytical treatment of all samples. JP participated in the design of the study, carried out molecular experiments and drafted the manuscript. JCL participated in the design of the study, performed histological analyses of all samples and participated in drafting the manuscript. PHMS, MH and MHAH participated in the design of the study and in drafting the manuscript. Additionally, MH performed statistical analyses. All authors read and approved the final manuscript.

\section{Author details}

'Laboratory of Molecular Diagnostics, Jeroen Bosch Hospital, PO Box 90153, 5200 ME 's-Hertogenbosch, The Netherlands. 'Laboratory of Pathology, Jeroen Bosch Hospital, PO Box 90153, 5223 GZ 's-Hertogenbosch, The Netherlands. ${ }^{3}$ Medical Microbiology and Infection Control, VU University Medical Center, PO Box 7057, 1007 MB Amsterdam, The Netherlands. ${ }^{4}$ Department Medical Microbiology, Maastricht University Medical Center, PO box 5800, 6202 AZ Maastricht, The Netherlands. ${ }^{5}$ Jeroen Bosch Academy, Jeroen Bosch Hospital, PO Box 90153, 5223 GZ 's-Hertogenbosch, The Netherlands.

Received: 29 December 2014 Accepted: 5 May 2015

Published online: 29 May 2015

\section{References}

1. Subramaniam DS, Isaacs C. Utilizing prognostic and predictive factors in breast cancer. Curr Treat Options Oncol. 2005;6(2):147-59.

2. Early Breast Cancer Trialists' Collaborative Group (EBCTCG). Effects of chemotherapy and hormonal therapy for early breast cancer on recurrence and 15-year survival: an overview of the randomised trials. Lancet 2005, 365(9472):1687-1717

3. Ring AE, Smith IE, Ashley S, Fulford LG, Lakhani SR. Oestrogen receptor status, pathological complete response and prognosis in patients receiving neoadjuvant chemotherapy for early breast cancer. Br J Cancer. 2004;91(12):2012-7.

4. Colleoni M, Viale G, Zahrieh D, Pruneri G, Gentilini O, Veronesi P, et al. Chemotherapy is more effective in patients with breast cancer not expressing steroid hormone receptors: a study of preoperative treatment. Clin Cancer Res. 2004;10(19):6622-8.

5. Coussens L, Yang-Feng TL, Liao YC, Chen E, Gray A, McGrath J, et al. Tyrosine kinase receptor with extensive homology to EGF receptor shares chromosomal location with neu oncogene. Science. 1985;230(4730):1132-9.

6. Yamamoto T, Ikawa S, Akiyama T, Semba K, Nomura N, Miyajima N, et al. Similarity of protein encoded by the human c-erb-B-2 gene to epidermal growth factor receptor. Nature. 1986;319(6050):230-4.

7. Tsai-Pflugfelder M, Liu LF, Liu AA, Tewey KM, Whang-Peng J, Knutsen T, et al. Cloning and sequencing of cDNA encoding human DNA topoisomerase II and localization of the gene to chromosome region 17q21-22. Proc Natl Acad Sci U S A. 1988;85(19):7177-81. 
8. Klapper LN, Kirschbaum MH, Sela M, Yarden Y. Biochemical and clinical implications of the ErbB/HER signaling network of growth factor receptors. Adv Cancer Res. 2000;77:25-79.

9. Alroy I, Yarden Y. The ErbB signaling network in embryogenesis and oncogenesis: signal diversification through combinatorial ligand-receptor interactions. FEBS Lett. 1997;410(1):83-6.

10. Tzahar E, Yarden Y. The ErbB-2/HER2 oncogenic receptor of adenocarcinomas: from orphanhood to multiple stromal ligands. Biochim Biophys Acta. 1998;1377(1):M25-37.

11. Bilous M, Ades C, Armes J, Bishop J, Brown R, Cooke B, et al. Predicting the HER2 status of breast cancer from basic histopathology data: an analysis of 1500 breast cancers as part of the HER2000 International Study. Breast. 2003;12(2):92-8.

12. Slamon DJ, Leyland-Jones B, Shak S, Fuchs H, Paton V, Bajamonde A, et al. Use of chemotherapy plus a monoclonal antibody against HER2 for metastatic breast cancer that overexpresses HER2. N Engl J Med. 2001;344(11):783-92.

13. Vogel CL, Cobleigh MA, Tripathy D, Gutheil JC, Harris LN, Fehrenbacher L, et al. Efficacy and safety of trastuzumab as a single agent in first-line treatment of HER2-overexpressing metastatic breast cancer. J Clin Oncol. 2002;20(3):719-26

14. Gennari A, Sormani MP, Pronzato P, Puntoni M, Colozza M, Pfeffer U, et al. HER2 status and efficacy of adjuvant anthracyclines in early breast cancer: a pooled analysis of randomized trials. J Natl Cancer Inst. 2008;100(1):14-20.

15. Press MF, Sauter G, Buyse M, Bernstein L, Guzman R, Santiago A, et al. Alteration of topoisomerase II-alpha gene in human breast cancer: association with responsiveness to anthracycline-based chemotherapy. J Clin Oncol. 2011;29(7):859-67.

16. Watt PM, Hickson ID. Structure and function of type II DNA topoisomerases. Biochem J. 1994:303(Pt 3):681-95.

17. Jarvinen TA, Tanner M, Rantanen V, Barlund M, Borg A, Grenman S, et al. Amplification and deletion of topoisomerase llalpha associate with ErbB-2 amplification and affect sensitivity to topoisomerase II inhibitor doxorubicin in breast cancer. Am J Pathol. 2000;156(3):839-47.

18. Konecny GE, Pauletti G, Untch M, Wang HJ, Mobus V, Kuhn W, et al. Association between HER2, TOP2A, and response to anthracycline-based preoperative chemotherapy in high-risk primary breast cancer. Breast Cancer Res Treat. 2010;120(2):481-9.

19. Binaschi M, Farinosi R, Borgnetto ME, Capranico G. In vivo site specificity and human isoenzyme selectivity of two topoisomerase Il-poisoning anthracyclines. Cancer Res. 2000;60(14):3770-6.

20. Binaschi M, Bigioni M, Cipollone A, Rossi C, Goso C, Maggi CA, et al. Anthracyclines: selected new developments. Curr Med Chem Anticancer Agents. 2001;1(2):113-30

21. Podo F, Buydens LM, Degani H, Hilhorst R, Klipp E, Gribbestad IS, et al. Triple-negative breast cancer: present challenges and new perspectives. Mol Oncol. 2010;4(3):209-29.

22. Reis-Filho JS, Tutt AN. Triple negative tumours: a critical review. Histopathology. 2008;52(1):108-18.

23. Bergamaschi A, Kim YH, Wang P, Sorlie T, Hernandez-Boussard T, Lonning $P E$, et al. Distinct patterns of DNA copy number alteration are associated with different clinicopathological features and gene-expression subtypes of breast cancer. Genes Chromosomes Cancer. 2006;45(11):1033-40.

24. Chin K, DeVries S, Fridlyand J, Spellman PT, Roydasgupta R, Kuo WL, et al. Genomic and transcriptional aberrations linked to breast cancer pathophysiologies. Cancer Cell. 2006;10(6):529-41.

25. Fridlyand J, Snijders AM, Ylstra B, Li H, Olshen A, Segraves R, et al. Breast tumor copy number aberration phenotypes and genomic instability. BMC Cancer. 2006;6:96

26. Hicks J, Krasnitz A, Lakshmi B, Navin NE, Riggs M, Leibu E, et al. Novel patterns of genome rearrangement and their association with survival in breast cancer. Genome Res. 2006;16(12):1465-79.

27. Loo LW, Grove DI, Williams EM, Neal CL, Cousens LA, Schubert EL, et al. Array comparative genomic hybridization analysis of genomic alterations in breast cancer subtypes. Cancer Res. 2004;64(23):8541-9.

28. Wang ZC, Lin M, Wei LJ, Li C, Miron A, Lodeiro G, et al. Loss of heterozygosity and its correlation with expression profiles in subclasses of invasive breast cancers. Cancer Res. 2004;64(1):64-71.

29. Yanagawa M, Ikemot K, Kawauchi S, Furuya T, Yamamoto S, Oka M, et al. Luminal A and luminal B (HER2 negative) subtypes of breast cancer consist of a mixture of tumors with different genotype. BMC Res Notes. 2012;5:376.
30. Ellsworth RE, Ellsworth DL, Patney HL, Deyarmin B, Love B, Hooke JA, et al. Amplification of HER2 is a marker for global genomic instability. BMC Cancer. 2008;8:297.

31. Munro AF, Twelves C, Thomas JS, Cameron DA, Bartlett JM. Chromosome instability and benefit from adjuvant anthracyclines in breast cancer. Br J Cancer 2012

32. Huijsmans CJ, Poodt J, Damen J, van der Linden JC, Savelkoul PH, Pruijt JF, et al. Single nucleotide polymorphism (SNP)-based loss of heterozygosity $(\mathrm{LOH})$ testing by real time PCR in patients suspect of myeloproliferative disease. PLoS One. 2012;7(7):e38362.

33. Arriola E, Marchio C, Tan DS, Drury SC, Lambros MB, Natrajan R, et al. Genomic analysis of the HER2/TOP2A amplicon in breast cancer and breast cancer cell lines. Lab Invest. 2008;88(5):491-503.

34. Kallioniemi OP, Kallioniemi A, Kurisu W, Thor A, Chen LC, Smith HS, et al. ERBB2 amplification in breast cancer analyzed by fluorescence in situ hybridization. Proc Natl Acad Sci U S A. 1992;89(12):5321-5.

35. Pauletti G, Godolphin W, Press MF, Slamon DJ. Detection and quantitation of HER-2/neu gene amplification in human breast cancer archival material using fluorescence in situ hybridization. Oncogene. 1996;13(1):63-72.

36. Persons DL, Borelli KA, Hsu PH. Quantitation of HER-2/neu and c-myc gene amplification in breast carcinoma using fluorescence in situ hybridization. Mod Pathol. 1997;10(7):720-7.

37. Carey LA, Dees EC, Sawyer L, Gatti L, Moore DT, Collichio F, et al. The triple negative paradox: primary tumor chemosensitivity of breast cancer subtypes. Clin Cancer Res. 2007;13(8):2329-34.

38. Huijsmans CJJ, Damen J, van der JC L, Savelkoul PHM, Hermans MHA. Comparative analysis of four methods to extract DNA from paraffin-embedded tissues: effect on downstream molecular applications. BMC Res Notes. 2010;3:239.

\section{Submit your next manuscript to BioMed Central and take full advantage of:}

- Convenient online submission

- Thorough peer review

- No space constraints or color figure charges

- Immediate publication on acceptance

- Inclusion in PubMed, CAS, Scopus and Google Scholar

- Research which is freely available for redistribution 